\title{
THE JANUARY MEETING ON FLUID DYNAMICS
}

The first program of the Committee on Fluid Dynamics of the American Physical Society was held at Columbia University on January 30, 31 and February 1, 1947. The American Mathematical Society acted as a cosponsor. These meetings of the Committee on Fluid Dynamics continue a series of closed meetings conducted during the war under the sponsorship of the Bureau of Ordnance of the U.S. Navy. The total attendance at the meeting was approximately three hundred. An audience survey on Thursday afternoon showed that the following 70 members of the American Mathematical Society were present:

Valentine Bargmann, Shepard Bartnoff, Stefan Bergman, Lipman Bers, Garrett Birkhoff, H. W. Bode, E. M. Boggs, T. E. Caywood, L. L. Cronvich, W. D. Duthie, Nat Edmonson, K. O. Friedrichs, W. J. Fry, Abe Gelbert, J. H. Giese, H. E. Goheen, N. A. Hall, Gerald Harrison, E. L. Haynes, A. D. Hestenes, J. H. Hett, Banesh Hoffmann, J. S. Isenberg, Aida Kalish, B. O. Koopman, P. A. Lagerstrom, Solomon Lefschetz, Joseph Lehner, B. A. Lengyel, Benjamin Lepson, C. C. Lin, D. P. Ling, Charles Loewner, E. R. Lorch, A. N. Lowan, J. K. L. MacDonald, A. K. Mitchell, Vladimir Morkovin, E. N. Nilson, G. H. Peebles, C. L. Pekeris, W. H. Pell, F. V. Pohle, Harry Polachek, Hillel Poritsky, William Prager, N. A. Renzetti, Daniel Resch, Moses Richardson, J. K. Riess, J. H. Rosenbloom, Arthur Sard, Hyman Serbin, Max Shiffman, S. S. Shü, Joseph Slepian, R. J. Slutz, T. L. Smith, George Springer, Benjamin Tannenbaum, A. H. Taub, J. E. Thompson, C. A. Truesdell, Y. W. Tschen, H. S. Tsien, J. W. Tukey, J. V. Wehausen, F. J. Weyl, H. A. Wood, A. W. Wundheiler.

Invited papers $F 1$ to $F 6$ were presented at the Thursday morning session, at which Professor H. W. Emmons presided. In the afternoon three invited addresses were given on aspects of Recent developments in compressible flow by Professors John von Neumann, G. F. J. Temple (of the Aeronautical Research Council of Great Britain) and Theodore von Kármán. Dr. H. L. Dryden presided.

Invited papers G1 to G6 were presented at the Friday morning session at which Dr. E. U. Condon presided. Contributed papers H1 to $\mathrm{H} 13$ were presented at the Saturday morning session at which Dr. R. J. Seeger presided.

Abstracts of all lettered papers except G4, G5 and G6 appeared in the Bulletin of the American Physical Society for January 30, 1947.

Of the 34 persons participating as speakers or chairmen, 9 were members of the American Mathematical Society.

F1. Rudolf Ladenburg: Interferometry of faster-than-sound phenomena.

F2. A. H. Taub: Refraction of shocks in ideal gases. 
F3. H. W. Liepmann: On the interaction between boundary layer and shock waves.

F4. Arthur Kantrowitz: The formation and stability of normal shock waves in channel flows.

F5. Francis Clauser: Notes on the thermodynamic propulsion of wind tunnels.

F6. W. F. Hilton: Variation of aerodynamic lift in the transonic speed range.

G1. Harold Wayland: Scale factors in water entry.

G2. Garrett Birkhoff and T. E. Caywood: Fluid flow patterns.

G3. R. W. Wood: The interaction of shock-waves, the spectra of cavity charge explosions, and the production of artificial meteors.

G4. H. W. Emmons: The solution of transonic fluid flow problems.

G5. C. C. Lin: Some recent investigations in hydrodynamic stability.

G6. J. W. Tukey: Linearization of solutions in supersonic flow.

H1. H. A. Einstein: On the hydraulic analogy between surface waves on a liquid and pressure waves in a compressible fluid.

H2. Valentine Bargmann: On nearly glancing reflection of shocks.

H3. J. H. Giese: Compressible flows with degenerate hodographs.

H4. Stefan Bergman: On an operator method in the theory of twodimensional flows of a compressible fluid.

H5. Zdenek Kopal: Some remarks on the limitations of linearized theory of supersonic flow around cones.

H6. F. L. Whipple: Supersonic problems in astronomy.

H7. R. N. Thomas: Comments on the motion of a solid body at high velocity through a compressible medium.

H8. H. K. Skramstad and G. B. Schubauer: Laminar boundarylayer oscillations and stability of laminar flow.

H9. Albert May and Jean C. Woodhull: Drag coefficient of steel spheres entering water.

H10. J. H. McMillan and E. N. Harvey: Drag and cavity of fast spheres shot into water.

H11. P. N. Metzelaar and D. E. Allmand: Impact pressure produced by flat plate striking water surface.

H12. B. L. Hicks: On the characterization of fields of diabatic flow.

H13. Y. H. Kuo: On the initial motion of a strong spherical shock.

J. W. TUKEY 